\title{
The reason for the Cosmological Constant Problem and a possible resolution
}

\author{
Kapil Chandra 1 \\ Department of physics, University of Bastar, Dharampura 1, Jagdalpur [CG], India, PIN - 494226
}

In this study we will show how quantum mechanics has failed to estimate the precise value of the cosmological constant because it uses the Planck mass/unit as the cut off. Since the expression for the Planck mass is numerically incorrect. This paper will show how a modification of the Planck unit gives a more accurate value and also fixes the long standing cosmological constant problem.

Keywords: vacuum energy density, cosmological constant problem, Planck unit.

Cosmological studied show that universe is expanding with increasing speed due to existence of some kind of unknown energy component [1]. We have no knowledge about this energy so that it is called dark energy content of universe. Our present theoretical understanding suggests that this energy content corresponds to the cosmological constant which is energy density of empty space itself. In this manuscript dark energy, vacuum energy density and cosmological constant terms used interchangeably.

Quantum mechanics $(\mathrm{QM})$ is one of the most successful theories in modern physics. It has passed many experimental test and suggests that the value of cosmological constant (CC) originate form the quantum fluctuations of vacuum. In order to estimate its value it must be summed over the zero-point energies of all normal modes of some field of mass $m$ up to a wave number cutoff $\Lambda>>m$ (with $h=c=l$ ) [2]. In this context the energy density of vacuum is as given below,

$$
\langle\rho\rangle=\int_{0}^{\Lambda} \frac{4 \pi k^{2} d k}{(2 \pi)^{3}} \frac{1}{2} \sqrt{k^{2}+m^{2}} \simeq \frac{\Lambda^{4}}{16 \pi^{2}}
$$

where $\langle\rho\rangle$ is energy density of empty space. If we take $\Lambda$ as Planck mass cut off, the predicted value is larger by over 120 orders of magnitude and this discrepancy is known as cosmological constant problem (CCP).

Many attempts to resolve this problem have been made and other proposed offer possible solutions. A summary of these attempts may be found somewhere in Ref. [3 - 7] to which interested readers are referred. The problem persists, however, so we reopen the investigation of quantum fluctuations in order to find out why it has failed to estimate the $\mathrm{CC}$ value precisely. To address this question we will use a different approach. Since, quantum theory is one of most successful theories of modern physics but in the case of $\mathrm{CC}$ it predicts an extreme value we reasoned that the value might be correct but uses an expression of Planck cutoff or Planck mass is not correct. Since it is dimensionally correct we argue that it might be a numerically incorrect expression.

This idea can be justified by studying the hypothesis which originates in the mathematical expression of this unit and may give us an understanding why this unit is numerically incorrect. Although, there are several method to derive this unit its a commonly know cat that all these derivations are based on a basic assumption or hypothesis that quantum and classical force/energy/physical entities are equal, comparable and interchangeable [8]. For example, consider the following relationship,

$$
\frac{h c}{R}=\frac{G m^{2}}{R}
$$

where all constants have their usual meanings and variable $R$ is space parameter.

The Eq. (2) will allow us to deduce the Planck mass but the quantum energy and gravitational potential are taken as equal i.e. classical and quantum energy are equal. The assumption that two different forms of energy are equal might be the reason that the expression for the Planck unit is dimensionally balanced but numerically not. We argue, it is wrong to incorporate the Planck and gravitational constant in

\footnotetext{
1 e-mail : kapil.chandra@gov.in
} 
this assumption. This is the reason that when this unit is used to estimate the value of vacuum energy density it gives its extreme value and cosmological constant problem emerges.

We propose following solution to this problem by correctly correlating the classical and quantum force. To do this we propose the following balance between classical and quantum force [9],

$$
\frac{h c}{R^{2}} \times \frac{m^{2} c^{3}}{h}=\frac{c^{4}}{G} \times G \frac{m^{2}}{R^{2}}
$$

all terms denotes different form of force and once we drop one terms from each side we see the correspondence to Eq. (2).

In this context the correct relation between classical and quantum force will be as written below,

$$
\frac{h c}{R^{2}}=\left(\frac{h c}{G m^{2}}\right) G \frac{m^{2}}{R^{2}}
$$

where one can take

$$
K=\frac{h c}{G m^{2}}
$$

if we take as a constant quantity by taking $m$ as mass of proton, its numerical value is nearly 39 orders of magnitude and this quantity is known as gravitational coupling constant.

The correct expression of Planck mass from Eq. (4) would now be,

$$
M_{p l}^{2}=\frac{h c}{\mathrm{GK}}
$$

and this will modify the mathematical expression of Planck mass and the other units and derived units.

Substituting this modified Planck mass/unit to Eq. (1) we get

$$
\langle\rho\rangle \simeq \frac{\Lambda^{4}}{16 \pi^{2} K^{2}}
$$

using this expression its numerical value is comparable to observed value of vacuum energy density. This suggests the cosmological constant problem or vacuum catastrophe is only due to hypothetical Planck unit and it appears that this problem has been unnecessarily emerged in quantum physics.
It is worthy to mention that Zeldovich used cut off $1 \mathrm{GeV}$ and got comparable value of vacuum energy but he did not explain why he took this limit [10]. This fact is clear here because the modified Planck mass is in this range i.e. the value of Eq. (6) is nearly in the same range; this might be an explanations for his idea.

In summary, quantum mechanics has failed to estimate the precise value of cosmological constant because it uses the Planck unit to compute its value and this unit is only dimensionally balanced but numerically not yielding its extreme large value. We can conclude that if the Planck unit is not really a correct expression then all predictions which are based on this unit must be incorrect as well. Further research recommended in this area.

\section{Acknowledgements}

I pay my hearty gratitude to the Dr. HS for his guidance. Sabah E Karam for this thought proving discussion on the topic related to cosmological constant problem and his support to prepare this manuscript.

\section{References:}

[1] A. Riess at el., Astron. J 116, 1009 (1999); S. Perlmutter at el., arXiv:astro-ph/9812133.

[2] R. Bousso, arXiv:1203.0307v2 [astro-ph.CO]; V. Sahni, arXiv:astro-ph/0202076v2.

[3] S. Weinberg, Rev. Mod. Phys. 61, 1 (1989).

[4] S. M. Carroll, W. H. Press, and E. L. Turner, Ann. Rev.Astron. Astrophys. 30, 499-542 (1992).

[5] S. Weinberg, Rev. Mod. Phys. 61, 1 (1989); S. Weinberg, arXiv:astro-ph/0005265; S. M. Carroll, W. H. Press, and E. L. Turner, Ann. Rev.Astron. Astrophys. 30, 499-542 (1992).

[6] A. Padilla, arXiv:1502:05296v1[hep-th]; A. D Dolgov, arXiv:astro-ph/9708045v1; S.M. Carroll, arXiv:astro-ph/0004075.

[7] Q. Wang, Z. Zhu, and W. G. Unruh, Phys. Rev. D 95, 103504 (2017) ; arXiv:1703.00543v2[gr-qc].

[8] M. Planck, S.-B. Preusse. Aka. Wiss., S.479-480 (1899).

[9] K. Chandra, JHEPGC, 5, 1098 (2019).

[10] Ya. B. Zeldovich, JETP Lett. 6, 316 (1967). 